\title{
Perfil epidemiológico de idosos submetidos à cirurgia de fratura de fêmur proximal em hospital escola na cidade de Marília, SP
}

\author{
Flávia Tiemi Yamamoto*, Sueli Moreira Pirolo**, Daniel Pereira Coqueiro***
}

\section{Resumo}

Estima-se que muitos idosos serão acometidos por trauma, tendo como consequência a fratura de fêmur proximal. Essa fratura apresenta altos índices de incapacidade funcional, diminuição da qualidade de vida, mortalidade e alto custo econômico. O objetivo deste estudo é caracterizar o perfil epidemiológico de idosos submetidos à correção cirúrgica de fratura de fêmur proximal. Trata-se de um estudo descritivo e epidemiológico realizado em hospital escola. Foram analisados 127 prontuários de idosos que se submeteram à correção cirúrgica dessa fratura entre 2012 e 2013, sendo a coleta guiada por roteiro com as variáveis: sexo, idade, estado civil, etnia, profissão, escolaridade, mecanismo do trauma, tipo de lesão, doenças associadas, tratamento cirúrgico, tempo de internação, complicações cirúrgicas e evolução. Os dados foram analisados por cálculo de frequências e variáveis de natureza quantitativa como média e mediana. $O$ teste-t de Student foi utilizado para comparação da média de idade em relação ao gênero dos pacientes. $O$ nível de significância $\alpha$ adotado foi $5 \%$. O perfil revelou prevalência de idosas, com idade média de 78 anos, etnia branca, viúvas, aposentadas, de baixa escolaridade e que apresentavam comorbidades. Predominou a fratura transtrocantérica, decorrente de queda da própria altura. O procedimento mais prevalente foi a osteossíntese, e a média hospitalar foi de 7,46 dias. A prótese de quadril foi procedimento com maior prevalência de complicação. O perfil sociodemográfico e do trauma dos idosos sinaliza a necessidade de melhor qualificar o tratamento para reduzir as complicações e o tempo de internação.

Palavras-chave: Fraturas do fêmur. Saúde do idoso. Epidemiologia.

\section{Introdução}

A legislação brasileira considera idosa aquela pessoa com idade igual ou superior a 60 anos. A previsão, diante do envelhecimento populacional, é de que em 2020 existam 1,2 bilhão de idosos no mundo, desses, 34 milhões serão indivíduos brasileiros, tornando o Brasil o sexto país no mundo em número de

* Enfermeira-residente do Programa de Residência Integrada Multiprofissional em Atenção Cirúrgica Especializada da Faculdade de Medicina de Marília, Marília, SP. Endereço para correspondência: Rua José Muller, 97. Bairro Jardim Bandeirantes. Cep: 17505-060. Marília, São Paulo. E-mail: flavinhati@hotmail.com

** Doutora em Enfermagem. Docente de Enfermagem Clínica da Faculdade de Medicina de Marília. E-mail: sueli.moreira@famema.br

*** $\quad$ Mestre em Ciências pela Universidade Federal de São Paulo - Escola Paulista de Medicina. Docente do curso de Educação Física da Faculdade de Ensino Superior do Interior Paulista, SP. E-mail: danicoq@hotmail.com

$\rightarrow$ http://dx.doi.org/10.5335/rbceh.v12i2.4791

Recebido em: 02/03/2015. Aceito em: 23/10/2015 
idosos (ENVELHECIMENTO..., 2005; MINAYO; COIMBRA JUNIOR, 2002).

$\mathrm{O}$ envelhecimento traz alterações fisiológicas nos sistemas musculoesquelético, circulatório, nervoso e sensorial, que, quando associadas a doenças crônico-degenerativas, interferem na segurança dos idosos, sendo que suas não compensações os tornam mais frágeis, levando a agravos, disfunção, dependência e quedas (SMELTZER; BARE, 2011).

A fratura de fêmur proximal encontra-se entre as lesões traumáticas mais comuns e graves em idosos. Classifica-se em intracapsular aquela que acomete o colo femoral, e em extracapsular a que acomete a região transtrocantérica e subtrocantérica. O tratamento é cirúrgico para a maioria das fraturas, podendo ser conservador em casos de fraturas incompletas ou sem desvio, acamados, sem condições de marcha ou quando o idoso possui comorbidades que contraindiquem a cirurgia (ROCHA; AZER; NASCIMENTO, 2009; SOUZA et al., 2007; MONTEIRO; FARO, 2006).

Após essa fratura, o idoso pode permanecer restrito por períodos prolongados de tempo, podendo levar à considerável incapacidade funcional, diminuição da independência, da qualidade de vida e, principalmente, redução na expectativa de vida. Em pesquisa realizada por Rocha, Azer e Nascimento (2009), quanto à evolução da fratura, somente $32,56 \%$ dos idosos readquiriram sua capacidade deambulatória prévia.

Em relação à taxa de mortalidade, Sakaki et al. (2004) apresentaram taxas médias em seu estudo de 5,5\% durante a internação hospitalar, $4,7 \%$ ao fim de um mês de seguimento, $11,9 \%$ com três meses, $10,8 \%$ com seis meses, $19,2 \%$ com um ano e $24,9 \%$ com dois anos, demonstrando que a fratura está relacionada a um elevado índice de mortalidade nessa população.

Assim, com embasamento no crescimento populacional, há estimativas de que, no século XXI, $40 \%$ dos idosos serão acometidos pelo trauma, tendo como consequência a fratura em $64 \%$ dos casos, dos quais, $62 \%$ será fratura de fêmur, podendo ser em região proximal, distal ou diáfise femoral (FABRICIO; RODRIGUES; COSTA JUNIOR, 2004; SOUZA; IGLESIAS, 2003). Em estudo, Silveira et al. (2005) referem que 6.500.000 indivíduos no mundo irão sofrer fratura de fêmur proximal no ano 2050 .

Esse tipo de fratura gera um alto custo econômico, devido à necessidade de hospitalização por um período variável, tratamentos cirúrgicos, cuidados intensivos no pós-operatório e programas de reabilitação por longos períodos (FERNANDES et al., 2011).

Desse modo, diante do envelhecimento populacional, de alta frequência de fratura de fêmur proximal, altas taxas de morbimortalidade com consequências biológicas, psicológicas e sociais para 0 idoso e sua família, e altos custos econômicos com tratamento e reabilitação, este estudo tem por objetivo caracterizar o perfil epidemiológico dos idosos que se submetem à cirurgia de correção da fratura de fêmur proximal.

A relevância desta investigação está na possibilidade de produzir informações sobre o adoecimento dos idosos em situação de fratura de fêmur, que podem subsidiar as equipes de saúde quanto aos 
cuidados de qualidade na prevenção e reabilitação desta morbidade, diminuindo assim os riscos de agravos e mortalidade.

\section{Método}

Trata-se de um estudo descritivo, epidemiológico e com abordagem quantitativa. A pesquisa foi submetida à apreciação do comitê de ética e pesquisa da Faculdade de Medicina de Marília para sua realização, sendo aprovada sob o protocolo $\mathrm{n}^{\mathrm{o}}$ 519.917, em 3 de fevereiro de 2014.

A investigação desenvolveu-se em uma unidade hospitalar de assistência e ensino, de referência para o serviço de ortopedia e traumatologia em uma área de abrangência de 62 municípios, com uma população estimada em 1.200.000 habitantes.

Participaram da pesquisa, 127 idosos que se submeteram ao procedimento cirúrgico de correção de fratura de fêmur proximal no período de $1^{\circ}$ de janeiro de 2012 a 31 de dezembro de 2013, identificados pelo Núcleo Técnico de Informação, setor do hospital responsável por manter o sistema de informação hospitalar.

A coleta de dados ocorreu a partir de fonte secundária, dos prontuários clínicos, para a qual foi utilizado um roteiro contendo as seguintes variáveis: sexo, idade, estado civil, etnia, profissão, escolaridade, mecanismo do trauma, tipo de lesão (colo, transtrocanteriana e subtrocanteriana), doenças associadas, tratamento cirúrgico, tempo de internação, complicações cirúrgicas e evolução. Essas variáveis foram adaptadas segundo estudos epidemiológicos em traumatologia (MUNIZ et al., 2007; ROCHA; AZER; NASCIMENTO, 2009). Esses dados foram coletados no serviço de arquivo da instituição com agenda prévia.
Após serem coletados, os dados foram inseridos em um banco de dados utilizando o programa de planilhas MS Excel ${ }^{\circledR}$. Os dados foram analisados por cálculo de frequências absolutas e relativas, e as variáveis de natureza quantitativa organizadas como média, mediana, desvio padrão. Foi utilizado o teste-t de Student para comparação da média de idade em relação ao gênero dos pacientes. O nível de significância $\alpha$ adotado foi igual a $5 \%$. Para as análises estatísticas foi utilizado o software Statistical Program for Social Sciences (SPSS ${ }^{\circledR}$ software, versão 20.0, Armonk, NY).

\section{Resultados}

\section{Perfil dos idosos}

Dos participantes, $23,6 \%$ eram do sexo masculino, com idade média de $76,8 \pm 8,2$ anos, e $76,4 \%$ eram do sexo feminino, com idade média de 78,7 \pm 7,84 anos. Observou-se que $16,5 \%$ dos pacientes tinham idade entre 60 e 69 anos, $38,6 \%$ entre 70 e 79 anos, $40,2 \%$ entre 80 e 89 anos, e apenas $4,7 \%$ dos pacientes tinham 90 anos ou mais. A média de idade não apresentou diferença estatisticamente significante $(p>0,05)$.

Tabela 1 - Frequência relativa e absoluta, idade média e desvio padrão dos pacientes segundo o gênero

\begin{tabular}{l|r|r}
\hline \multicolumn{1}{c|}{ Gênero* } & \multicolumn{1}{c|}{$\mathrm{n}(\%)$} & Idade $($ média $\pm \mathrm{dp})$ \\
\hline Feminino & $97(76,4)$ & $78,70 \pm 7,84$ \\
Masculino & $30(23,6)$ & $75,87 \pm 8,21$ \\
Total & $127(100,0)$ & $78,03 \pm 7,99$ \\
\hline
\end{tabular}

Fonte: Prontuários clínicos, Marília, 2014.

Nota: * teste-t de Student. 
Em relação ao estado civil, enquanto $31 \%$ tinham companheiros, $69 \%$ não os tinham. A frequência de pacientes aposentados ou pensionistas foi de $89,3 \%$, enquanto $10,7 \%$ dos pacientes relataram exercer ativamente uma profissão. Desses, $83,5 \%$ eram de etnia branca. Quanto à escolaridade, $59,7 \%$ tinham o ensino fundamental, $35,5 \%$ eram analfabetos e somente $4,8 \%$ apresentaram ensino médio ou superior completo (Tabela 2).

Tabela 2 - Frequência relativa e absoluta das variáveis sociodemográficas dos pacientes com fratura de fêmur

\begin{tabular}{l|r|r}
\hline \multicolumn{1}{c|}{ Variáveis } & $\begin{array}{c}\text { Pacientes } \\
(\mathrm{n}=127)\end{array}$ & \multicolumn{1}{c}{$\%$} \\
\hline Idade & 21 & 16,5 \\
60 a 69 & 49 & 38,6 \\
70 a 79 & 51 & 40,2 \\
80 a 89 & 06 & 4,7 \\
90 ou mais & & \\
Estado civil & 87 & 69,0 \\
Sem companheiro(a) & 39 & 31,0 \\
Com companheiro(a) & & \\
Etnia & 106 & 83,5 \\
Branco & 13 & 10,2 \\
Pardo & 07 & 5,5 \\
Negro & 01 & 0,8 \\
Asiático & & \\
Escolaridade & 44 & 35,5 \\
Analfabeto & 74 & 59,7 \\
Ensino fundamental & 03 & 2,4 \\
Ensino médio & 03 & 2,4 \\
Ensino superior & & \\
Condição econômica & 10 & 10,7 \\
Ativo & 83 & 89,3 \\
Aposentado/pensionista &
\end{tabular}

Fonte: Prontuários clínicos, Marília, 2014.

Quanto aos tipos, a principal fratura observada foi a transtrocantérica, em $58,3 \%$ dos pacientes, enquanto as fraturas colo femorais acometeram $33,9 \%$ dos avaliados, e as subtrocantéricas, 7,9\% dos casos. As lesões transtrocantéricas foram as mais frequentes no gênero feminino, com 62 casos $(63,9 \%)$, e no gênero masculino, as lesões colo femorais foram as mais frequentes, com 16 casos (53,3\%).

Em relação ao mecanismo do trauma, houve predominância de casos decorrentes de queda da própria altura $(95,3 \%)$. Quedas de escadas, da cama e de andaimes representaram apenas $4,7 \%$. Quanto às comorbidades, 92,1\% dos pacientes apresentaram alguma (Tabela 3); desses, 38,6\% tinham três ou mais doenças crônicas, $32,3 \%$ duas doenças, $21,3 \%$ apenas uma, e 7,9\% não apresentaram nenhuma doença crônica.

A escolha da técnica cirúrgica varia de acordo com o tipo de fratura e leva em consideração o nível de atividade do idoso. Neste estudo, a fratura transtrocantérica e a subtrocantérica foram submetidas à osteossíntese, sendo os métodos mais utilizados o Dynamic Hip Screw (DHS), com 48,8\%, seguido pelo Proximal Femoral Nail (PFN), com 17,3\%. Quanto à fratura do colo de fêmur, o principal método empregado foi a artroplastia total de quadril, com $13,4 \%$, seguido pela artroplastia parcial de quadril, com $11 \%$ (Tabela 3 ).

Desses procedimentos, $21,3 \%$ apresentaram complicações cirúrgicas, sendo as três mais prevalentes a infecção de sítio cirúrgico (33\%), a trombose venosa profunda (15\%) e o tromboembolismo pulmonar (11\%). O maior índice de complicação ocorreu nas próteses de quadril $(32,2 \%)$ quando comparadas com as osteossínteses $(17,7 \%)$.

Pode-se observar que $37 \%$ dos pacientes ficaram internados por um período menor que cinco dias, e $44,1 \%$ permaneceram internados por um período entre cin- 
co e dez dias. A mediana do tempo entre a admissão no hospital e a cirurgia foi de dois dias, e o tempo médio foi $3,24( \pm 4,56)$ dias (mínimo de zero dia e máximo de 34 dias). A média do tempo de internação foi de seis dias, e o tempo de internação médio foi de 7,46 $( \pm 5,77)$ dias (mínimo de dois dias e máximo de 36 dias).

Tabela 3 - Frequência relativa e absoluta das variáveis clínicas dos idosos

\begin{tabular}{l|r|r}
\hline \multicolumn{1}{c|}{ Variáveis } & Pacientes & \multicolumn{1}{|c}{$\%$} \\
\hline Mecanismo do trauma & 121 & \\
Queda da própria altura & 3 & 95,3 \\
Queda da escada & 2 & 1,4 \\
Queda da cama & 1 & 0,8 \\
Queda do andaime & & \\
Doenças associadas & 10 & 7,9 \\
Nenhuma & 27 & 21,3 \\
Uma & 41 & 32,3 \\
Duas & 49 & 38,6 \\
Três ou mais & & \\
Tipo de tratamento cirúrgico* & 62 & 48,8 \\
DHS & 22 & 17,3 \\
PFN & 3 & 2,4 \\
DCS & 6 & 4,7 \\
Parafuso canulado & 3 & 2,4 \\
DHS + parafuso canulado & 14 & 11,0 \\
Prótese parcial & 17 & 13,4 \\
Prótese total & & \\
Complicações cirúrgicas & 27 & 21,3 \\
Sim & 100 & 78,7 \\
Não & & \\
Tempo de internação & 47 & 37,0 \\
< 5 dias & 56 & 44,1 \\
5-10 dias & $17-15$ dias & 13,4 \\
16-20 dias & 2,4 \\
> 20 dias & 3,1 \\
\hline Pro & & \\
\hline
\end{tabular}

Fonte: Prontuários clínicos, Marília, 2014.

Nota: *DHS - Dynamic Hip Screw; PFN - Proximal Femoral Nail; DCS - Dynamic Condilar Screw.
$\mathrm{Na}$ avaliação da mobilidade em último registro, $25,2 \%$ dos pacientes deambulavam sem auxílio, $33,1 \%$ necessitavam de auxílio para deambulação, e $24,4 \%$ não deambulavam. O nível de mortalidade encontrado foi de $10,2 \%$, e não retornaram para a consulta pós-operatória $7,1 \%$.

\section{Discussão}

O perfil sociodemográfico dos idosos observados indica um predomínio de mulheres idosas, com idade média geral de $78,03 \pm 7,99$ anos, de etnia branca, sem companheiro, em grande parte devido ao falecimento do parceiro, aposentadas ou pensionistas, de baixa escolaridade, que já apresentavam uma ou mais patologias e sofreram queda.

A prevalência do gênero feminino também foi encontrada em estudos realizados por Muniz et al. (2007) e por Martini et al. (2012). Esses autores justificam que a maior incidência de fraturas de fêmur proximal nessa população deve-se à maior prevalência de doenças crônicas, a uma maior exposição às atividades domésticas e uma menor presença de quantidade de massa magra e de força muscular em relação aos homens, assim como incidência de osteoporose, que ocorre durante o climatério, além de sinalizar a feminização do envelhecimento.

A idade média geral aponta para um maior índice de fraturas a partir dos 70 anos de idade, sendo que a idade média para a fratura no gênero masculino foi menor em relação ao feminino. Hungria Neto, Dias e Almeida (2011) referem que homens sofrem maiores índices de fra- 
turas relacionados aos traumas de alta energia quando ativos, e por esse motivo tendem a sofrer fraturas em idades inferiores as das mulheres.

A etnia branca encontrada pode ser explicada pelas diferenças existentes nas dimensões e na densidade óssea do fêmur em relação às raças branca e negra. A raça negra tem ossos mais densos devido ao maior pico de massa óssea que ganham durante a maturação esquelética do osso, além de ter uma diferente dimensão do fêmur em comparação com a raça branca, que é definida como uma proteção geométrica do quadril, traduzindo-se por um menor comprimento do eixo do quadril, maior largura do colo do fêmur e um ângulo cervico diafisário aumentado (KEATING, 2013). Outro fator relacionado à etnia se refere ao fato de as raças branca e amarela apresentarem maior incidência de osteoporose do que indivíduos das raças negra e parda (CURY; ZACCHELLO, 2007).

O principal mecanismo de trauma foi queda da própria altura. Segundo Pereira et al. (2002), essa ocorrência é definida como o deslocamento não intencional do corpo para um nível inferior à posição inicial com incapacidade de correção em tempo hábil, determinado por circunstâncias multifatoriais que comprometem a estabilidade. Dentre essas circunstâncias estão os fatores extrínsecos, relacionados a questões sociais e ambientais, e os fatores intrínsecos, que são decorrentes de alterações fisiológicas associadas ou não a condições patológicas crônicas e ao concomitante uso de medicamentos (COSTA et al., 2012).
Quanto ao fator extrínseco, esta pesquisa identificou que grande parte dos idosos não tinha a presença de um companheiro, sendo que este fato determina uma maior prevalência de quedas, fato explicado pelo cuidado mútuo existente entre parceiros (SIQUEIRA et al., 2007).

A abordagem da condição econômica nesse contexto também tem relevância, visto que modificações na infraestrutura ambiental e uso de objetos de proteção pessoal contra quedas demandam condições financeiras que muitos idosos não têm.

Quanto aos fatores intrínsecos, a grande maioria dos idosos tinha ao menos uma doença crônica, sendo prevalentes idosos que apresentaram três ou mais doenças associadas à fratura de fêmur proximal. A presença de doenças crônicas predispõe os idosos a um maior risco de quedas e consequentemente fraturas, uma vez que evoluem com algum grau de perda da capacidade funcional que se soma às alterações fisiológicas do envelhecimento, além de tornar os idosos usuários de grande quantidade de medicações que alteram o grau de atenção, as respostas motoras e fisiológicas (COSTA et al., 2012).

O perfil dos idosos frente ao trauma e sua evolução possibilitou identificar a prevalência da fratura transtrocantérica, sendo a osteossíntese o procedimento cirúrgico mais utilizado, e as artroplastias são os procedimentos cirúrgicos que apresentaram maior índice de complicações.

A prevalência da fratura transtrocantérica encontra-se em conformidade com a literatura e é justificada por tratar-se de uma região constituída em 
sua maior parte por osso esponjoso, que com o envelhecimento tem sua morfologia modificada, ocorrendo um espessamento da camada cortical e aumento do diâmetro do osso, tornando-o mais frágil (RUSSELL, 2013; MUNIZ et al., 2007; MATOS et al., 2010).

Quanto ao procedimento cirúrgico, tanto a osteossíntese quanto as artroplastias apresentam seus benefícios e riscos. A osteossíntese tem menor tempo de duração cirúrgica, menor perda de sangue e menor risco de infecção profunda, o que diminui o trauma cirúrgico. No entanto, a literatura traz achados diferentes deste estudo por considerar que as artroplastias têm um número menor de revisão cirúrgica e complicações, sendo mais funcional e de menor risco de reoperação para os idosos (LUSTOSA; BASTOS, 2009).

O tipo de fratura e a escolha da técnica cirúrgica determinam o material utilizado para a fixação cirúrgica. Em consequência, acarreta o tempo previsto para início da deambulação, a descarga de peso no membro e as restrições em alguns movimentos. Deve-se considerar a importância do ortostatismo e da deambulação precoce na prevenção de complicações inerentes ao imobilismo, como as encontradas neste estudo.

Prevenir as complicações no pós-operatório pressupõe o acompanhamento de um profissional de saúde para realização de exercícios e início da deambulação pós-operatória, pois esses cuidados não somente previnem complicações como aumentam a força muscular, melhoram a segurança e eficiência da deambulação, contribuindo com ganhos funcionais e ge- rando confiança e condições emocionais positivas, visto que grande parte dos idosos que caem e fraturam o fêmur adquirem medo de deambular novamente e sofrer nova queda (CARNEIRO; ALVES; MERCADANTE, 2013; FABRICIO; RODRIGUES; COSTA JUNIOR, 2004).

Quanto ao tempo de internação, este estudo demonstrou que o tempo médio de internação antes da cirurgia ultrapassa 0 preconizado por Takata e Basile (2008), que recomendam que essa cirurgia deve ocorrer nas primeiras 48 horas, já que a postergação leva invariavelmente à piora das condições e do quadro clínico do paciente, aumentando a morbidade e a mortalidade.

Segundo Cunha et al. (2008), o atraso ou o adiamento da cirurgia, muitas vezes, é causado por problemas burocráticos, referentes à disponibilidade de salas cirúrgicas e de especialistas na área. Esse atraso, geralmente, está relacionado à instabilidade clínica dos idosos, levando a um maior período entre a fratura e a cirurgia (GRIMES et al., 2002).

\section{Considerações finais}

Este estudo possibilitou caracterizar o perfil sociodemográfico, o trauma e seus tratamentos em idosos que sofreram fratura de fêmur proximal, além de identificar a necessidade de investimentos para qualificar o cuidado ao diminuir as complicações e o tempo de internação dos pacientes. Contudo, o período amostral de dois anos traz apenas em parte a realidade dos idosos em condição de fratura de fêmur proximal. 
A busca de informações no prontuário clínico foi oportuna, pois permitiu o acesso a dados relevantes para caracterizar o perfil dos idosos. Os resultados apontam para a necessidade de novas pesquisas epidemiológicas, com a intenção de produzir indicadores de saúde relacionados ao envelhecimento.

Os limites encontrados nesta pesquisa foram a falta de algumas informações nos prontuários dos idosos pesquisados, como a descrição da queda e da evolução dos casos, uma vez que foi considerado somente o último registro sem levar em conta o tempo pós-operatório. Esses dados são importantes para as prevenções de queda e o tratamento da fratura de fêmur proximal, permitindo um cuidado além do curativo.

\section{Epidemiological profile of the elderly undergoing proximal femur fracture sugery in hospital school in the city of Marília, SP}

\section{Abstract}

It is estimated that many seniors will be affected by trauma that may result in the proximal femur fracture. This fracture shows high rates of functional incapacity, decrease quality of life, mortality and high economic cost. The study objective to characterize the epidemiology of elderly undergoing surgical correction of proximal femur fracture. This is a descriptive and epidemiological study in a hospital school. Were analyzed 127 medical records of seniors who underwent surgical correction of this fracture between 2012 and 2013, with the collection guided by script with the variables: gender, age, marital status, ethnicity, occupation, education, mecha- nism of trauma, injury, associated diseases, surgical treatment, length of stay, surgical complications and evolution. The data were analyzed by calculation of frequency and quantitative nature of variables organized as the mean, median and standard deviation. The T-test of Student was used for comparison of average age in relation to the gender of the patients. The $\alpha$ significance level adopted was 5\%. The profile revealed prevalence of elderly female, with an average age of 78 years, white ethnicity, widows, pensioners, low educational level and with comorbidities. The most observed fracture was the transtrochanteric, most due to fall of their own time, being the most prevalent procedure the osteosynthesis and the average 7.46 hospital days. The prosthesis of hip procedure with higher prevalence of complications. The sociodemographic profile of trauma and the elderly signals the need to better qualify the treatment to reduce complications and length of hospital stay.

Keywords: Fractures of the femur. Health oh the Elderly. Epidemiology.

\section{Referências}

CARNEIRO, M. B.; ALVES, D. P. L.; MERCADANTE, M. T. Fisioterapia no pós-operatório de fratura proximal do fêmur em idosos. Revisão da literatura. Acta Ortopédica Brasileira, São Paulo, v. 21, n. 3, p. 175-178, maio-jun. 2013.

COSTA, I. C. P. et al. Fatores de risco de quedas em idosos: produção científica em periódicos online no âmbito da saúde. $R e$ vista Brasileira de Ciências da Saúde, João Pessoa, v. 16, n. 3, p. 445-452, 2012.

CUNHA, P. T. S. et al. Fratura de quadril em idosos: tempo de abordagem cirúrgica e sua associação quanto à delirium e infecção. Acta Ortopédica Brasileira, São Paulo, v. 16, n. 3, p. 173-76, 2008. 
CURY, A. F.; ZACCHELLO, K. P. Osteoporose: prevalência e fatores de risco em mulheres de clínica privada maiores de 49 anos de idade. Acta Ortopédica Brasileira, São Paulo, v. 15, n. 3, p. 146-150, 2007.

ENVELHECIMENTO ativo: uma política de saúde. Tradução de Suzana Gontijo. Brasília, DF: Organização Pan-Americana da Saúde, 2005.

FABRICIO, S. C. C.; RODRIGUES, R. A. P.; COSTA JUNIOR, M. L. Causas e consequências de quedas em idosos atendidos em Hospital Público. Revista de Saúde Pública, São Paulo, v. 38, n. 1, p. 93-99, fev. 2004.

FERNANDES, R. A. et al. Fraturas de fêmur proximal no idoso: estudo de custo da doença sob a perspectiva de um hospital público do Rio de Janeiro, Brasil. Physis: Revista de Saúde Coletiva, Rio de Janeiro, v. 21, n. 2, p. 395-416, 2011.

GRIMES, J. P. et al. The effects of time-tosurgery on mortality and morbidity in patients following hip fracture. The American Journal of Medicine, Tucson, v. 112, n. 9, p. 702-709, June 2002.

HUNGRIA NETO, J. S.; DIAS, C. R.; ALMEIDA, J. D. B. Características epidemiológicas e causas da fratura do terço proximal do fêmur em idosos. Revista Brasileira de Ortopedia, São Paulo, v. 46, n. 6, p. 660-667, 2011.

KEATING, J. Fratura do colo do fêmur. In: BULCHOLZ, R. W. et al. (Ed.) Fraturas em adultos de Rockwood e Green. 7. ed. Barueri: Manole, 2013. p. 1561-1592. v. 2.

LUSTOSA, L. P; BASTOS, E. O. Fraturas proximais do fêmur em idosos: qual o melhor tratamento? Acta Ortopédica Brasileira, São Paulo, v. 17, n. 5, p. 309-312, 2009.

MARTINI, F. P. et al. Estudo epidemiológico das fraturas de fêmur proximal em Canoas no ano de 2010. Revista da AMRIGS, Porto Alegre, v. 56, n. 4, p. 320-324, 2012.

MATOS, M. A. et al. Avaliação intra-hospitalar de pacientes portadores de fraturas de fêmur proximal. Revista Baiana de Saúde Pública, Salvador, v. 34, n. 1, p. 30-35, 2010.

MINAYO, M. C. S.; COIMBRA JUNIOR, C. E. A. Antropologia, saúde e envelhecimento. [on-line]. Rio de Janeiro: Fiocruz, 2002. 209 p. (Coleção Antropologia \& Saúde). Disponível em: <http://static.scielo.org/scielobooks/ d2frp/pdf/Mhttp://static.scielo.org/scielobooks/d2frp/pdf/minayo -978857541043. pdfinayo-9788575413043.pdf $>$. Acesso em: 10 jan. 2015.

MONTEIRO, C. R.; FARO, A. C. M. O cuidador do idoso e sua compreensão sobre a prevenção e o tratamento cirúrgico das fraturas de fêmur. Revista de Estudos Interdisciplinares sobre o Envelhecimento, Porto Alegre, v. 10, p. 105-121, dez. 2006.

MUNIZ, C. F. et al. Caracterização dos idosos com fratura de fêmur proximal atendidos em hospital escola público. Revista Espaço para a Saúde, Londrina, v. 8, n. 2, p. 33-38, jun. 2007.

PEREIRA, S. R. M. et al. Quedas em idosos. In: JATENE, F. B. et al. Projeto diretrizes. São Paulo: Associação Médica Brasileira e de Brasília; Conselho Federal de Medicina. 2002. p. 405-414. v. 1.

ROCHA, M. A.; AZER, H. W.; NASCIMENTO, V. G. Evolução funcional nas fraturas da extremidade proximal do fêmur. Acta Ortopédica Brasileira, São Paulo, v. 17, n. 1, p. 17-21, 2009.

RUSSELL, T. A. Fraturas intertrocantéricas. In: BULCHOLZ, R. W. et al. (Ed.). Fraturas em adultos de Rockwood e Green. 7. ed. Barueri: Manole, 2013. p. 1596-1637. v. 2.

SAKAKI, M. H. et al. Estudo da mortalidade na fratura do fêmur proximal em idosos. Acta Ortopédica Brasileira, São Paulo, v. 12, n. 4, p. 242-249, out.-dez. 2004.

SILVEIRA, V. A. L. et al. Incidência de fratura do quadril em área urbana do Nordeste brasileiro. Cad. Saúde Pública, Rio de Janeiro, v. 21, n. 3, p. 907-912, maio-jun. 2005. 
SIQUEIRA, F. V. et al. Prevalência de quedas em idosos e fatores associados. Revista de Saúde Pública, São Paulo, v. 41, n. 5, p. 749-756, out. 2007.

SMELTZER, S. C.; BARE, B. G. Cuidado de saúde do idoso. In: . Tratado de enfermagem médico cirúrgico. 11. ed. Rio de Janeiro: Guanabara Koogan, 2011. p. 142-161.

SOUZA, J. A. G.; IGLESIAS, A. C. R. Trauma no Idoso. Revista da Associação Médica Brasileira, São Paulo, v. 1, n. 48, p. 79-86, jan.-mar. 2003.

SOUZA, R. C. et al. Aplicação de medidas de ajuste de risco para a mortalidade após fratura proximal de fêmur. Revista de Saúde Pública, São Paulo, v. 41, n. 1, p. 625-631, ago. 2007.

TAKATA, E. T.; BASILE, R. Fratura de fêmur proximal. In: FALOPPA, F.; ALBERTONI, W. M. Ortopedia e traumatologia. Barueri: Manole, 2008. p. 567-574. 